\title{
Heterogeneity of local tissue microenvironment influences differentiation of oligodendroglial progenitors
}

\author{
Joanna Sypecka, Anna Sarnowska \\ NeuroRepair Department, Mossakowski Medical Research Centre, Polish Academy of Sciences, Warsaw, Poland
}

\begin{abstract}
Glial NG2-positive precursors are considered as suitable candidates for the cell-based therapies for disorders characterised by depletion of functional oligodendrocytes and ongoing hypo/demyelination. They are known to be among the first cells to react to CNS dysfunction. Their fate could be however considerably affected by the heterogeneity of the local tissue microenvironment. To address this issue, a comparison of mRNA expression of the crucial trophic factors was carried out in organotypic slices from the hippocampus (as a well-recognised neurogenic area) and from the spinal cord (where gliogenic signals are assumed to predominate). The molecular analysis revealed substantial differences in the mRNA levels of main factors governing cell biology. Additionally, the ability of these two microenvironments to promote the astrocytic differentiation of oligodendroglial progenitors was assessed as well. While the GFAP+ cells constituted the very minor population in control experiments, their amount increased significantly during culturing in the medium continuously conditioned by either hippocampal or the spinal cord slices. The observed susceptibility to the influence of the local extracellular signals might efficiently contribute to the reported glial scar formation. It presumably could also modify the fate of the endogenous or transplanted precursors, what should be taken into consideration in cell-based therapies.
\end{abstract}

Key words: oligodendroglial progenitors, glia, astrocytes, organotypic slices, hippocampus, spinal cord, trophic factors, heterogeneity, microenvironment.

\section{Introduction}

Oligodendrocyte progenitors (OPCs) are NG2-positive cells abundantly populating the young and adult central nervous system (CNS). They are capable of myelinogenesis, but they are also among the first cells to react to CNS dysfunction [32]. They are known to be affected in many inherited $[15,44,54]$ and acquired disorders, both acute like spinal cord injury and brain trauma $[1,6,49]$ and chronic like intoxication or immuno- logical diseases [39]. The resulting depletion of functional oligodendrocytes accompanied by ongoing hypo/ demyelination leads to an urgent need for cell replacement therapies based on either recruitment of endogenous progenitors or cell transplantation.

Endogenous OPCs are generated during perinatal gliogenesis and remain scattered across white and grey matter in large quantities $[26,36,52]$. In adult CNS, they could also be derived from neural stem cells inhabiting the subventricular zone (SVZ) $[10,28]$ and the hilus 
of the dentate gyrus (DG), a part of the hippocampal non-neurogenic area [23]. Thus, there is a natural and abundant reservoir of cells existing within CNS, which could be mobilized for neurorepair purposes. In the case of inherited diseases leading to oligodendrocyte malfunction and myelin disturbances, the cell transplantation would presumably be the best solution. However, the fate of both recruited endogenous precursors and the transplanted cells is considerably affected by the local tissue microenvironment, composed of a cocktail of active compounds like trophic factors, cytokines, morphogenes and extracellular matrix components $[18,22,40]$. Over the past decade, the neurogenic potential of glia-committed precursors has been intensively investigated. In our previous reports, the ability of the neonatal OPCs to express the spectrum of pro-neuronal markers in the microenvironment created by hippocampal slices was shown. A fraction of the labelled OPCs seeded onto the slices, which migrated and integrated to the tissue, adopted the neuronal phenotype [45]. The results have also revealed that the neurogenic effect could be exerted by paracrine signalling. This points to the presence of soluble active compounds governing cell commitment.

Our observations together with reports coming from other laboratories lead to a question concerning differences in the composition of nervous tissue derived from distinct CNS regions $[2,34,46,50]$. To address this issue, organotypic slices from the hippocampus (as a well-recognised neurogenic area) and from the spinal cord (where gliogenic signals are assumed to predominate) were subjected to a molecular analysis of those selected factors which regulate crucial cell processes. Additionally, the ability of these two microenvironments to promote the astrocytic differentiation was studied to verify the postulated multipotency of the oligodendroglial progenitors.

\section{Material and methods \\ Oligodendrocyte progenitors isolation and culturing}

Oligodendrocyte progenitors were isolated from a mixed primary glial culture, established from brain hemispheres of neonatal Wistar Cmd:(WI)WU rats. The procedure was approved by the Local Ethics Committee on Animal Care and Use. The detailed procedure is described elsewhere $[22,45]$. Briefly, the method is based on sequentially dislodging separate glial fractions (first microglia and subsequently oligodendrocyte pre- cursors, while astrocytes remain strongly adhered to plastic) [24] from a 2-week-old primary culture. The shaken-off progenitors were spun down and the resulting pellet was mechanically dispersed with a 22- $\mu \mathrm{m}$ needle in serum-free standard DMEM medium, supplemented with a solution of antibiotics. The cell suspension was purified from contaminating non-oligodendroglial cells by filtration through $80 \mu \mathrm{m}$ and subsequently through $41-\mu \mathrm{m}$ Millipore membranes. The additional step of the OPC purification was based on the poor adhesion of the progenitors to the uncoated plastic, while the other glial fractions stick to the plastic surface within 2-3 hours. After collecting of the supernatant containing floating OPCs, the cells were seeded onto poly-L-lysine-coated

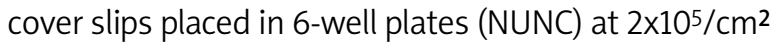
density and cultured for the subsequent 10 DIV in serum-free culture medium.

\section{Organotypic hippocampal culture (OHC)}

A hippocampal organotypic culture was established from the brains of 7-day-old Wistar rats according to the previously described method [38,42]. All the procedures of animal handling practices were accepted by the Local Ethics Committee on Animal Care and Use. Briefly, the anesthetized animals were decapitated and their brains were carefully isolated and inserted into ice-cold HBSS (Gibco) buffer. The 400- $\mu \mathrm{m}$ thin hippocampal slices, cut by a Mcllwain tissue chopper, were placed onto Millicell-CM (Millipore) membranes and inserted in 6-well plates (NUNC). The slices were then cultured in a defined medium composed of DMEM 50\%, HEPES, HBSS 25\%, horse serum 25\% (Gibco), 2 mmol/l L-glutamine, $5 \mathrm{mg} / \mathrm{ml}$ glucose, $1 \%$ amphotericin $B$ and $0.4 \%$ penicillin-streptomycin. The serum concentration in the culture medium was gradually lowered and from the 7th DIV onwards, the slices were cultured in serum-free conditions. On the $8^{\text {th }}$ DIV the organotypic slices were used for co-culture experiments.

\section{Organotypic spinal cord culture (OSC)}

The organotypic longitudinal spinal cord slice culture was established according to the procedure described elsewhere [19]. The spinal cords were extracted from the same animals used for isolating the hippocampi. After dissection, $10 \mathrm{~mm}$ longitudinal sagittal slices of the spinal cord were cut using a tissue chopper (Mcllwain) at a thickness of $350 \mu \mathrm{m}$ and transferred onto a permeable Millicell-CM (4 slices per membrane) and cultured according to the protocol used for $\mathrm{OHC}$. 


\section{Co-culturing of OPCs with organotypic slices (OHC/OSC)}

The purified OPC fraction was left to adhere to polyL-lysine-coated cover slips for approximately $1 \mathrm{~h}$ and was used for setting up the co-culture experiments with either the spinal cord or the hippocampal organotypic slices. The Millicell-CM membranes containing the slices were transferred to the plates with OPCs and co-cultured in indirect contact (Fig. 1) for the next 5 days in serum-free DMEM supplemented with an AAS antibiotic solution (Gibco). The culture medium was changed every second day. On the 5 DIV, the organotypic slices were gently removed from the membranes with an

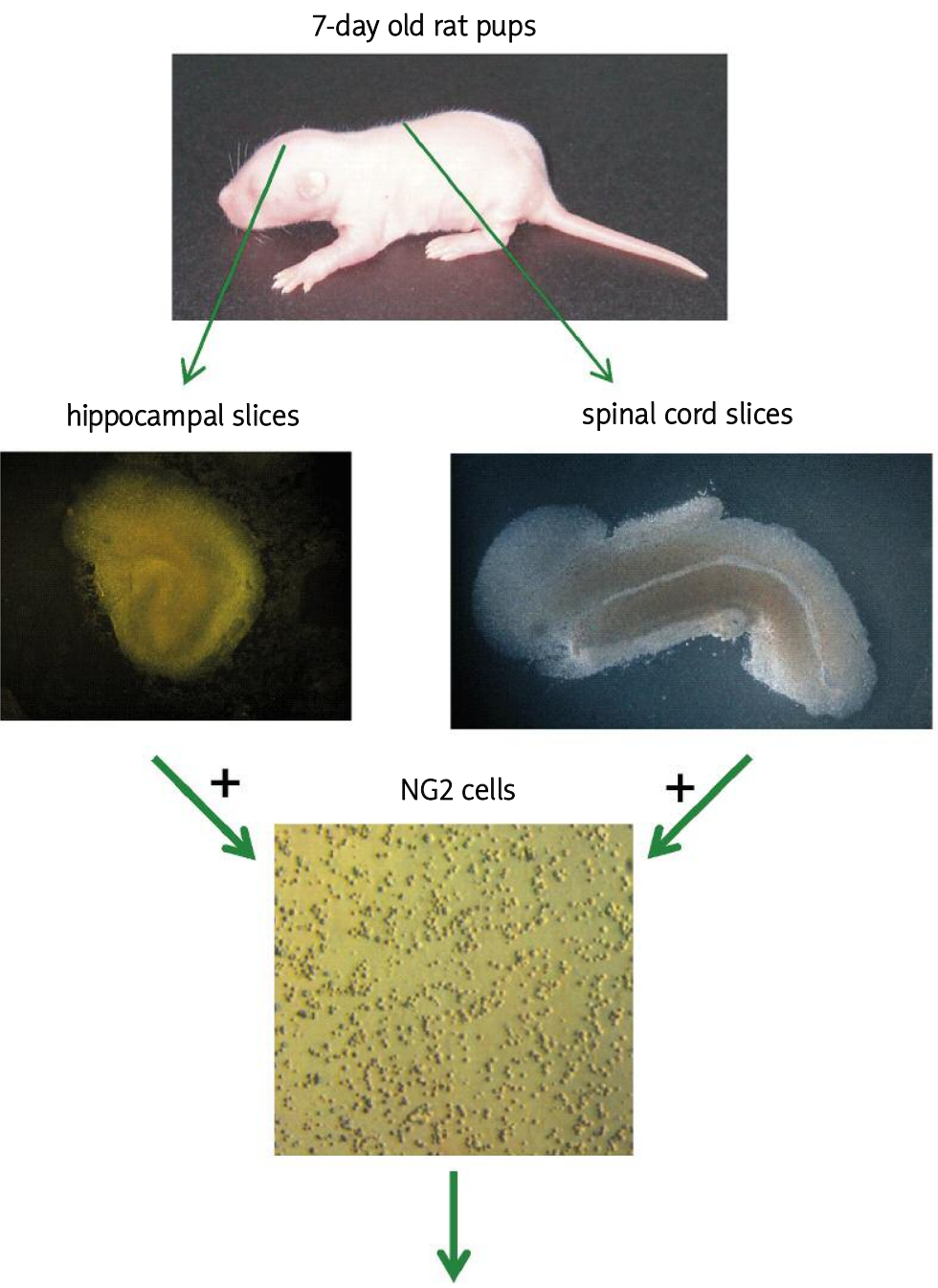

NG2/organotypic slice co-culture

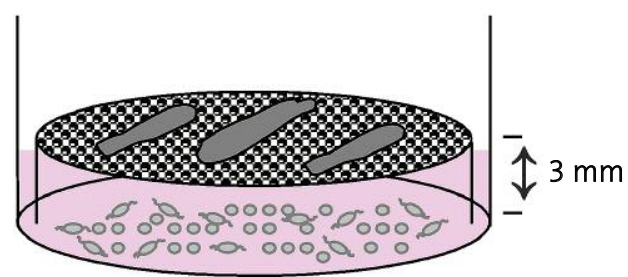

Fig. 1. The schematic diagram of the co-culture experiments. The hippocampal and spinal cord slices were prepared from the same 7-day-old rat and cultured on the cell culture inserts, serving for the indirect coculture with rat neonatal NG2 precursors. 
ultra-thin brush, put into RNAlater Solution (Applied Biosystems) and stored at $4^{\circ} \mathrm{C}$ until the nucleic acid had been extracted.

\section{Reverse transcription and quantitative real-time RT-PCR analysis}

In order to extract total RNA, the Trizol reagent (Invitrogen) was applied and the purified samples were subjected to reverse transcription reaction by application of High Capacity RNA-to-cDNA Kit (Applied Biosystems), following the manufacturer's instructions. To measure the selected trophic factors (including neurotrophins) and cytokines, the commercial RT2 Profiler $^{\mathrm{TM}}$ PCR Array for rat neurotrophins (SABioscience) was applied according to the supplier's protocol. The samples containing 50, 75 and 100 ng of CDNA were amplified using SYBR Green PCR Master Mix (Applied Biosystems) and ABI Prism 7500 Sequence Detection System. Each sample was tested in triplicate during three analyses and the parameters selected for the performed PCR reactions were as follows: $2 \mathrm{~min}$ at $50^{\circ} \mathrm{C}, 10 \mathrm{~min}$ at $95^{\circ} \mathrm{C}$, 40 cycles of $15 \mathrm{~s}$ at $95^{\circ} \mathrm{C}$ and $1 \mathrm{~min}$ at $60^{\circ} \mathrm{C}$. To determine the PCR product specificity, a dissociation curve was plotted and $1.5 \%$ agarose gel electrophoresis was performed to check the amplicon size. The obtained data were normalized against that of the $\beta$-actin gene and threshold cycle values $(\Delta \mathrm{Ct})$ were quantified as fold changes by the $2^{-\Delta \Delta C t}$ method [20].

\section{Immunocytochemical analysis}

The cell commitment and differentiation were assessed by staining the PFA-fixed cells with a panel of specific antibodies. To ensure the specificity of binding, the cells were immersed in $10 \%$ normal goat serum in PBS in at $25^{\circ} \mathrm{C}$ for an hour and then the primary antibodies were applied overnight. The stages of oligodendrocyte maturation were verified by applying the following: rabbit polyclonal anti-PDGF $\alpha$ R (Santa Cruz) and anti-NG2 (Chemicon) - for the precursor state; monoclonal anti-mouse against 04 (Sigma), CNP-ase (Sigma) and GalC (Chemicon) - for immature oligodendrocytes; monoclonal anti-mouse anti-MBP (Sigma) for myelinating cells. To identify the generated astrocytes, rabbit polyclonal antibody against GFAP (Dako) was used. Appropriate secondary antibodies, conjugated to either Alexa-488 or Alexa-546 (Molecular Probes), were applied for 1 hour at RT. Before immersing in Fluoromont, the cell nuclei were stained with $5 \mu \mathrm{m}$ Hoechst 33258 (Sigma). The labelled cells were examined using an Axio- vert 25 fluorescence microscope (Zeiss). Four randomly selected fields on each slide were taken by a Videotronic CCD-4230 camera and a cell count was done.

\section{Statistical analysis}

The data obtained due to molecular and immunocytochemical examinations were subjected to a statistical analysis with one-way analysis of variance (ANOVA) followed by the Bonferroni's Multiple Comparison Test. All the values were expressed as mean \pm SEM, ${ }^{*} p<0.05$.

\section{Results}

To assess the ability of the oligodendroglial precursor to acquire the astrocytic phenotype in various microenvironment conditions, a purified cell population from neonatal rat brains was co-cultured with organotypic slices derived from distinct CNS regions. Before establishing the OPC co-cultures, both the spinal cord and hippocampal slices were deprived of serum supplement to prevent the cell from being stimulated by additional, undefined compounds. Subsequently, an indirect coculture system (Fig. 1) was set up to study the influence of the soluble factors released from the organotypic slices into the culture medium on OPCs differentiating into astrocytes. Simultaneously, the OPCs were allowed to spontaneously differentiate in the same, serum-free medium as the control in order to evaluate the potential stimulating effect exerted by the CNS slices. The data obtained as a result of immunocytochemical analysis showed that $99.2 \pm 0.92 \%$ of OPCs had matured from NG2 antigen expressing cells (Figs. 2A-C) into $\mathrm{O} 4$ and GalC-positive oligodendrocytes during the 5-day - in vitro period. Another interesting finding concerned the promotion of astroglial differentiation by slightly elevating the serum content in the culture media. By adding $1 \%$ serum, the number of GFAP-positive cells in the differentiating population increased by $2.48 \pm 0.8 \%$.

The examination of how the cells differentiated in the co-cultures with organotypic slices led to some more interesting observations. While the $\mathrm{GFAP}^{+}$cells constituted a very minor population in control experiments, their amount increased as much as $18.5 \pm 2.31 \%$ of the total cell number in the medium continuously conditioned by either hippocampal or the spinal cord slices (Figs. 2D-F). A quantitative analysis of both coculture types revealed approximately comparative (15$19 \%)$ content of astrocytes with no statistically significant differences. 

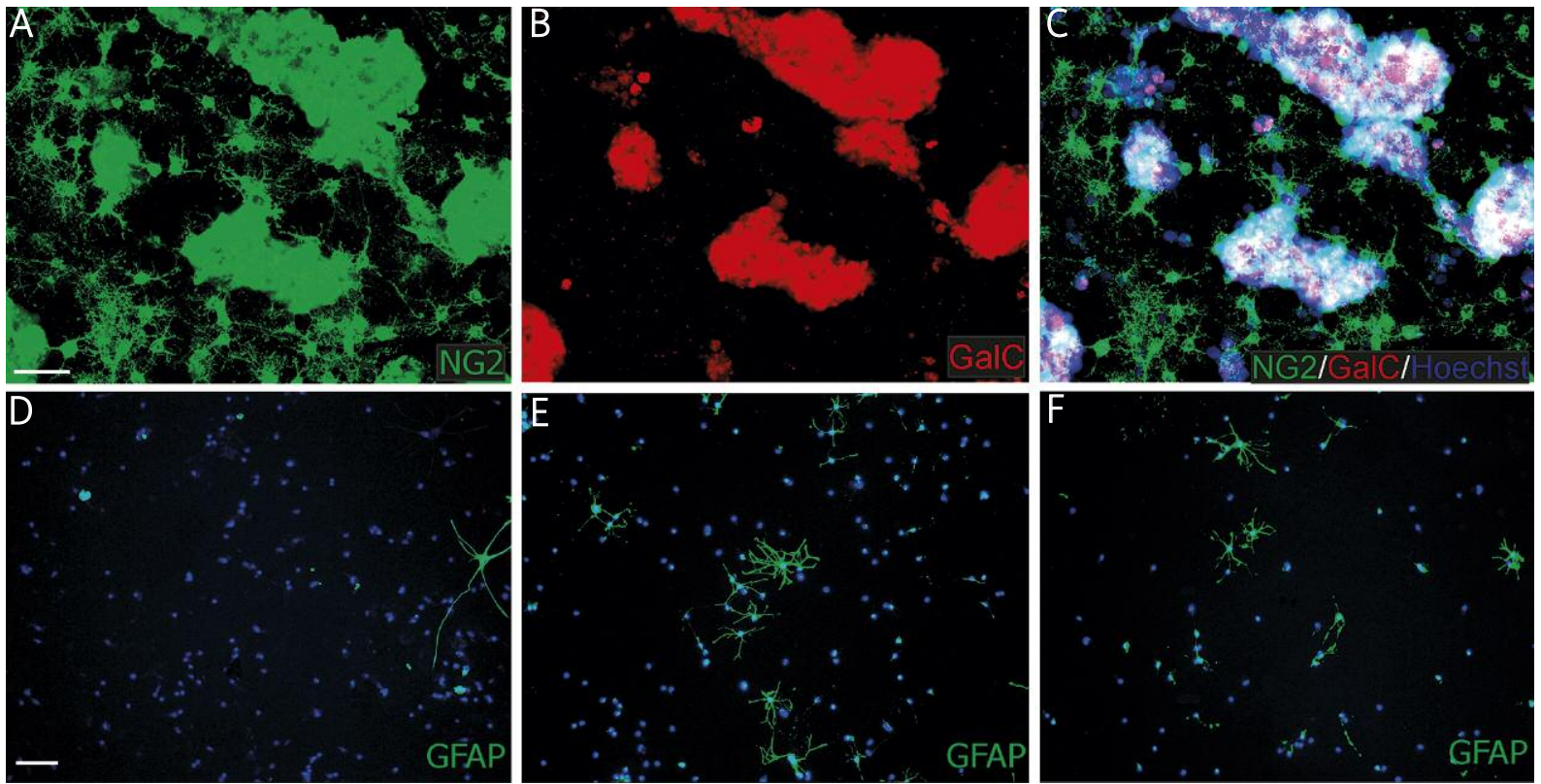

Fig. 2. Differentiation of oligodendroglial precursors in serum and supplement-free conditions during 5 DIV. A, B, C) Immunostaining of control oligodendrocytes with classical markers: NG2 (green) and GalC (red). D, E, F) Astroglial phenotype acquired differentiating control OPCS (D), and co-cultured with hippocampal (E) and spinal cord (F) organotypic slices. Cells are identified by GFAP (green) expression. Cell nuclei are visualized by staining with Hoechst 33258 dye (blue). Scale bar $=50 \mu \mathrm{m}$.

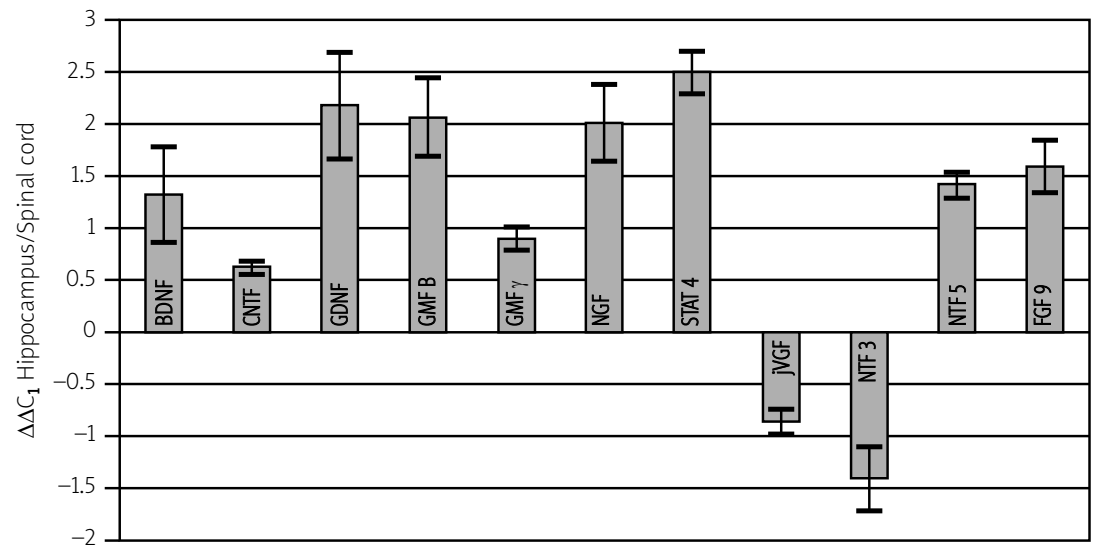

Fig. 3. Relative expression of neurotrophin mRNA in hippocampal versus spinal cord slices, estimated by real-time PCR quantification. All values are expressed as $\Delta \Delta C_{t}$ calculated against $\beta$-actin and are significant at least at ${ }^{*} p<0.05$.

The observed potent capability of the paracrine signals provided by organotypic slices to trigger astroglial differentiation prompted us to check the expression of the selected cytokines and trophic factors at the mRNA level by means of semi-quantitative molecular methods. The microarray screening for neurotrophins and related factors which are known to be engag- ed in main cellular functions revealed a significantly elevated expression of all of the examined factors in organotypic slices in relation to housekeeping genes (B-actin, ribosomal protein L13A, lactate dehydrogenase $\mathrm{A}$ ). In turn, a comparison between hippocampal and spinal cord slices showed that out of the 15 analysed factors the expression of nine in the hippocampal 
slices was noticeably elevated, two were downregulated and only the other four were similarly expressed (Fig. 3). The factors, of which the mRNA expression is elevated, include neurotrophins such as brain-derived trophic factor (BDNF), nerve growth factor (NGF), ciliary neurotrophic factor (CNTF), glial-derived neurotrophic factor (GDNF) and other factors too, such as neurotrophin-5 (NTF5), fibroblast growth factor 9 (FGF 9), Signal Transducer and Activator of Transcription protein (STAT4) and glial maturation factor (GMF, isoforms $\beta$ and $\gamma$ ). The factors, which are strongly expressed in the spinal cord, are VGF nerve growth factor inducible protein and neurotrophin 3 (NTF3). Those without noticeably changed expression include leukemia inhibitory factor (LIF) as well as STAT1, STAT2 and STAT3.

\section{Discussion}

Over the past decade, oligodendrocyte disorders accompanied by myelin deficit, malformation or its loss have become targets for cell-based therapies. Oligodendrocyte progenitors may be most conveniently used for the complex neurorepair strategies. First of all, they are already glia-commitment progenitors themselves, which considerably reduces the danger of neoplasia and assures their differentiating into myelinating cells $[4,12,16,24,43,51]$. Moreover, there is a growing list of evidence that OPCs exhibit some important properties of neural stem cells in a conducive microenvironment. They have been shown to be sensitive to being stimulated by the external clues and prove to be multipotential. However, even in the highly stimulus milieu, part of the OPC population is still proliferative.

The enumerated properties could be beneficial to the process of restoring a damaged tissue. While promoting neurogenesis is usually highly desirable, the ability of differentiating into astrocytes might however prove be a serious obstacle. Many CNS injuries are associated with the glial scar formation, which significantly hinders precursors from migrating and delivering anti-inflammatory and trophic factors $[9,37,41,50]$.

The objective of our studies was to assess the ability of OPCs to acquire the astroglial phenotype in a microenvironment created by the nervous tissue derived from distinct CNS regions. While in controls, all of the progenitors matured into oligodendrocytes expressing myelin components, the medium continuously conditioned by either the spinal cord or hippocampal slices triggered astroglial differentiation of a part of the cultured OPCs. What has been known for a long time is that astrocytes can be derived from common glial precursors in vitro while being cultured in high concentrations of serum $[14,30,31,47]$. This observation contributed to one of the pioneer terms for describing oligodendrocyte progenitors: O-A2. In our serum free co-culture system with the organotypic slices (i.e. with the preserved tissue organization) the paracrine signals proved to be potent to force the astroglial commitment of OPCs. There are also reports describing a similar in vivo effect, found in different CNS regions $[33,56]$. The observation coming from our study concerning the promotion of astroglial differentiation in cocultures with hippocampal and spinal cord slices are in agreement with the reports about the glial scar formation in injuries occurring to the brain and spinal cord.

The heterogeneity of the nervous tissue microenvironment, suggested by the results obtained in our and other laboratories, prompted us to analyse a spectrum of active compounds that are known to regulate the main biological functions like proliferation, survival, commitment and differentiation. A comparison of data gathered from molecular studies showed that certain crucial factors involved in important biological processes occurring in cells are differently expressed. The expression of nine examined factors (out of fifteen) turned out to be increased in the hippocampal slices when compared to the spinal cord slices, two others were down-regulated, while only four were similarly expressed. These findings prove conclusively that the local tissue microenvironment within the nervous system is heterogenic.

What is worth noting is that among factors being highly expressed in the hippocampus are those exerting the neurotrophic effects: neurotrophins NGF and BDNF [5] as well as CNTF [3,27] and GDNF [7,57]. They supposedly play a role in recruiting new neurons, protecting them and regenerating nerves $[13,57]$. GMFs were shown to be involved in regulating glia activity and in enhancing the pro-inflammatory responses in a range of selected diseases [53]. Signal Transducer and Activator of Transcription together with FGF-2 are engaged in regulating genes responsible for cell proliferation, migration, differentiation, and survival, as well as for neurogenesis and axon growth [8,35]. Up-regulation of those selected genes is presumably associated with the neurogenic regions characteristic of hippocampus.

The specificity of different CNS regions, indicated by the results obtained in the presented study, might 
significantly modify cell commitment and differentiation. Since therapies based on mobilizing endogenous progenitors or on transplanting stem cells are intensely pursued $[11,17,21,29,48]$, the cell fate in various local microenvironments should be investigated for the purpose of predicting the outcome of the applied treatment.

In conclusion, the presented work shows the substantial differences in the expression of the factors governing the crucial processes involved in oligodendroglial precursors biology between organotypic slices derived from distinct CNS regions. The stated local tissue heterogeneity should be taken into consideration when planning cell-based therapies.

\section{Acknowledgements}

We thank Mrs Hanna Winiarska and Mr Lukasz Strojek for excellent technical assistance. This work is supported by the Polish Ministry of Science and Higher Education grant 0345/B/P01/2010/38 and 05728/B/NZ4/ 2011/01.

\section{References}

1. Almad A, Sahinkaya FR, McTigue DM. Oligodendrocyte fate after spinal cord injury. Neurotherapeutics 2011; 8: 262-273.

2. Banu MA, Alter R, Boockvar JA. Resetting the stem cell clock: impact of the systemic milieu on oligodendrocyte precursor cell differentiation and remyelination. Neurosurgery 2012; 70: N15-17.

3. Blanchard J, Chohan MO, Li B, Liu F, Iqbal K, Grundke-Iqbal I. Beneficial effect of a CNTF tetrapeptide on adult hippocampal neurogenesis, neuronal plasticity, and spatial memory in mice. J Alzheimers Dis 2010; 21: 1185-1195.

4. Cao Q, He Q, Wang Y, Cheng X, Howard RM, Zhang Y, DeVries WH, Shields CB, Magnuson DS, Xu XM, Kim DH, Whittemore SR. Transplantation of ciliary neurotrophic factor-expressing adult oligodendrocyte precursor cells promotes remyelination and functional recovery after spinal cord injury. I Neurosci 2010; 24 : 2989-3001.

5. Chao MV, Rajagopal R, Lee FS. Neurotrophin signalling in health and disease. Clin Sci (Lond) 2006; 110: 167-173.

6. Dewar D, Underhill SM, Goldberg MP. Oligodendrocytes and ischemic brain injury. J Cerebral Blood Flow \& Metabolism 2003; 23: 263-274.

7. Duarte EP, Curcio M, Canzoniero LM, Duarte CB. Neuroprotection by GDNF in the ischemic brain. Growth Factors 2012; 30: 242-257.

8. Fortin D, Rom E, Sun H, Yayon A, Bansal R. Distinct fibroblast growth factor (FGF)/FGF receptor signaling pairs initiate diverse cellular responses in the oligodendrocyte lineage. J Neurosci 2005; 25: 7470-7479

9. Frontczak-Baniewicz M, Chrapusta SJ, Sulejczak D. Long-term consequences of surgical brain injury - characteristics of the neurovascular unit and formation and demise of the glial scar in a rat model. Folia Neuropathol 2011; 49: 204-218.
10. Gonzalez-Perez O, Alvarez-Buylla A. Oligodendrogenesis in the subventricular zone and the role of epidermal growth factor. Brain Res Rev 2011; 67: 147-156.

11. Gójska-Grymajło A, Nyka WM, Zieliński M, Jakubowski Z. CD34/ CXCR4 stem cell dynamics in acute stroke patients. Folia Neuropathol 2012; 50: 140-146.

12. Gornicka-Pawlak el B, Janowski M, Habich A, Jablonska A, Drela K, Kozlowska H, Lukomska B, Sypecka J, Domanska-Janik K. Systemic treatment of focal brain injury in the rat by human umbilical cord blood cells being at different level of neural commitment. Acta Neurobiol Exp (Wars) 2011; 71: 46-64.

13. Harvey AR, Ooi JW, Rodger J. Neurotrophic factors and the regeneration of adult retinal ganglion cell axons. Int Rev Neurobiol 2012; 106: 1-33.

14. Hu JG, Zhang YX, Qi Q, Wang R, Shen L, Zhang C, Xi J, Zhou JS, Lu HZ. Expression of BMP-2 and BMP-4 proteins by type- 1 and type-2 astrocytes induced from neural stem cells under different differentiation conditions. Acta Neurobiol Exp (Wars) 2012; 72: 95-101.

15. Huyghe A, Horzinski L, Hénaut A, Gaillard M, Bertini E, Schiffmann R, Rodriguez D, Dantal Y, Boespflug-Tanguy O, Fogli A. Developmental splicing deregulation in leukodystrophies related to EIF2B mutations. PLoS One 2012; 7: e38264.

16. Ishii A, Furusho M, Bansal R.Sustained Activation of ERK1/2 MAPK in Oligodendrocytes and Schwann Cells Enhances Myelin Growth and Stimulates Oligodendrocyte Progenitor Expansion. J Neurosci 2013; 33: 175-186.

17. Karimi-Abdolrezaee S, Schut D, Wang J, Fehlings MG. Chondroitinase and growth factors enhance activation and oligodendrocyte differentiation of endogenous neural precursor cells after spinal cord injury. PLoS One 2012; 7: e37589

18. Kim H, Cooke MJ, Shoichet MS. Creating permissive microenvironments for stem cell transplantation into the central nervous system. Trends Biotechnol 2012; 30: 55-63.

19. Sypecka J, Sarnowska A, Gadomska-Szabłowska I, Lukomska B, Domanska-Janik K. Differentiation of glia-committed NG2 cells: the role of factors released from hippocampus and spinal cord. Acta Neurobiologiae Experimentalis (Wars) 2013; 73 [Epub ahead of print].

20. Livak KJ, Schmittgen TD. Analysis of relative gene expression data using real-time quantitative PCR and the 2(-Delta Delta C(T)) Method. Methods 2001; 25: 402-408.

21. Lü HZ, Wang YX, Zou J, Li Y, Fu SL, Jin JQ, Hu JG, Lu PH. Differentiation of neural precursor cell-derived oligodendrocyte progenitor cells following transplantation into normal and injured spinal cords. Differentiation 2010; 80: 228-240.

22. Markiewicz I, Sypecka J, Domanska-Janik K, Wyszomirski T, Lukomska B. Cellular environment directs differentiation of human umbilical cord blood-derived neural stem cells in vitro. J Histochem Cytochem 2011; 59: 289-301.

23. Matsumoto Y, Tsunekawa Y, Nomura T, Suto F, Matsumata M, Tsuchiya S, Osumi N. Differential proliferation rhythm of neural progenitor and oligodendrocyte precursor cells in the young adult hippocampus. PLoS One 2011; 6: e27628.

24. McCarthy KD. de Vellis J. Preparation of separate astroglial and oligodendroglial cell cultures from rat cerebral tissue. J Cell Biol 1980; 85: 890-902.

25. Mi S, Miller RH, Tang W, Lee X, Hu B, Wu W, Zhang Y, Shields CB, Zhang Y, Miklasz S, Shea D, Mason J, Franklin RJ, Ji B, Shao Z, 
Chédotal A, Bernard F, Roulois A, Xu J, Jung V, Pepinsky B. Promotion of central nervous system remyelination by induced differentiation of oligodendrocyte precursor cells. Ann Neurol 2009; 65: 304-315.

26. Miller FD, Gauthier AS. Timing is everything: making neurons versus glia in the developing cortex. Neuron 2007; 54: 357-369.

27. Müller S, Chakrapani BP, Schwegler H, Hofmann HD, Kirsch M Neurogenesis in the dentate gyrus depends on ciliary neurotrophic factor and signal transducer and activator of transcription 3 signaling. Stem Cells 2009; 27: 431-441.

28. Nait-Oumesmar B, Picard-Riéra N, Kerninon C, Baron-Van Evercooren $\mathrm{A}$. The role of SVZ-derived neural precursors in demyelinating diseases: from animal models to multiple sclerosis. J Neurol Sci 2008; 265: 26-31

29. Naganska E, Matyja E. Amyotrophic lateral sclerosis - looking for pathogenesis and effective therapy. Folia Neuropathol 2011; 49 1-13.

30. Noble M, Murray K, Stroobant P, Waterfield MD, Riddle P. Plateletderived growth factor promotes division and motility and inhibits premature differentiation of the oligodendrocyte/type-2 astrocyte progenitor cell. Nature 1988; 333: 560-562.

31. Raff MC, Miller RH, Noble M. A glial progenitor cell that develops in vitro into an astrocyte or an oligodendrocyte depending on culture medium. Nature 1983; 303: 390-396.

32. Robel S, Berninge B, Götz M. The stem cell potential of glia: les sons from reactive gliosis. Nature Reviews Neuroscience 201; 12 : 88-104.

33. Rompani SB, Cepko CL. A common progenitor for retinal astrocytes and oligodendrocytes. J Neurosci. 2010; 30: 4970-4980.

34. Rosenberg SS, Kelland EE, Tokar E, De la Torre AR, Chan JR. The geometric and spatial constraints of the microenvironment induce oligodendrocyte differentiation. Proc Natl Acad Sci U S A 2008; 105: 14662-14667.

35. Reuss B, von Bohlen und Halbach O. Fibroblast growth factors and their receptors in the central nervous system. Cell Tissue Res 2003; 313: 139-157.

36. Rowitch DH, Kriegstein AR. Developmental genetics of vertebrate glial-cell specification. Nature 2010; 468: 214-222.

37. Sabo JK, Cate HS. Signalling pathways that inhibit the capacity of precursor cells for myelin repair. Int J Mol Sci 2013; 4: 1031-1049.

38. Sarnowska A, Braun H, Sauerzweig S, Reymann KG. The neuroprotective effect of bone marrow stem cells is not dependent on direct cell contact with hypoxic injured tissue. Exp Neurol 2009; 215: 317-327.

39. Saulsbury MD, Heyliger SO, Wang K, Johnson DJ. Chlorpyrifos induces oxidative stress in oligodendrocyte progenitor cells. Toxicology 2009; 259: 1-9.

40. Sim FJ, Windrem MS, Goldman SA. Fate determination of adult human glial progenitor cells. Neuron Glia Biol 2009; 5: 45-55.

41. Son JL, Soto I, Oglesby E, Lopez-Roca T, Pease ME, Quigley HA Marsh-Armstrong N. Glaucomatous optic nerve injury involves early astrocyte reactivity and late oligodendrocyte loss. Glia 2010; 58: 780-789.

42. Stoppini L, Buchs PA, Muller D. A simple method for organotypic cultures of nervous tissue. J Neurosci Methods 1991; 37: 173-182.

43. Sypecka J. Searching for oligodendrocyte precursors for cell replacement therapies. Acta Neurobiol Exp (Wars) 2011; 71: 94-102.
44. Sypecka J, Domańska-Janik K. Phenotypic diversity resulting from a point mutation. Folia Neuropathol 2006; 44: 244-250.

45. Sypecka J, Sarnowska A, Domanska-Janik K. Crucial role of the local micro-environment in fate decision of neonatal rat NG2 progenitors. Cell Prolif 2009; 42: 661-671.

46. Tamura Y, Kataoka Y, Cui Y, Takamori Y, Watanabe Y, Yamada H. Multi-directional differentiation of doublecortin- and NG2immunopositive progenitor cells in the adult rat neocortex in vivo. Eur J Neurosci 2007; 25: 3489-3498.

47. Tanner DC, Cherry JD, Mayer-Pröschel M. Oligodendrocyte progenitors reversibly exit the cell cycle and give rise to astrocytes in response to interferon- $\gamma$. J Neurosci 2011; 31: 6235-6246.

48. Tep C, Lim TH, Ko PO, Getahun S, Ryu JC, Goettl VM, Massa SM, Basso M, Longo FM, Yoon SO. Oral Administration of a Small Molecule Targeted to Block proNGF Binding to p75 Promotes Myelin Sparing and Functional Recovery after Spinal Cord Injury. J Neurosci 2013; 33: 397-410.

49. Volpe JJ, Kinney HC, Jensen FE, Rosenberg PA. The developing oligodendrocyte: key cellular target in brain injury in the premature infant. Int J Dev Neurosci 2011; 29: 423-440.

50. Wang Y, Cheng X, He Q, Zheng Y, Kim DH, Whittemore SR, Cao QL Astrocytes from the contused spinal cord inhibit oligodendrocyte differentiation of adult oligodendrocyte precursor cells by increasing the expression of bone morphogenetic proteins. J Neurosci 2011; 31: 6053-6058.

51. Watson RA, Yeung TM. What is the potential of oligodendrocyte progenitor cells to successfully treat human spinal cord injury? BMC Neurol 2011; 11: 113.

52. Wegner M. A matter of identity: transcriptional control in oligodendrocytes. J Mol Neurosci 2008; 35: 3-12.

53. Zaheer S, Thangavel R, Wu Y, Khan MM, Kempuraj D, Zaheer A. Enhanced expression of glia maturation factor correlates with glial activation in the brain of triple transgenic Alzheimer's disease mice. Neurochem Res 2013; 38: 218-225.

54. Zdaniuk G, Wierzba-Bobrowicz T, Szpak GM, Stępień T. Astroglia disturbances during development of the central nervous system in fetuses with Down's syndrome. Folia Neuropathol 2011; 49: 109-114.

55. Zhang L, Ma Z, Smith GM, Wen X, Pressman Y, Wood PM, Xu XM. GDNF-enhanced axonal regeneration and myelination following spinal cord injury is mediated by primary effects on neurons. Glia 2009; 57: 1178-1191.

56. Zhu X, Hill RA, Nishiyama A. NG2 cells generate oligodendrocytes and gray matter astrocytes in the spinal cord. Neuron Glia Biol 2008; 4: 19-26.

57. Zuo T, Qin JY, Chen J, Shi Z, Liu M, Gao X, Gao D. Involvement of $\mathrm{N}$-cadherin in the protective effect of glial cell line-derived neurotrophic factor on dopaminergic neuron damage. Int J Mol Med 2013; doi: 10.3892/ijmm.2013.1226. 